\title{
Effect of Fluoroethylene Carbonate Additive on the Initial Formation of Solid Electrolyte Interphase on Oxygen Functionalized Graphitic Anode in Lithium Ion Batteries
}

\author{
Nadia N. Intan $\uparrow$ and Jim Pfaendtner* $+\dagger$ \\ $\dagger$ Department of Chemical Engineering, University of Washington, Seattle, Washington 98195 \\ \$ Physical Sciences Division, Pacific Northwest National Laboratory, Richland, Washington 99352 \\ *Email: jpfaendt@uw.edu
}

\begin{abstract}
The formation of a solid electrolyte interphase (SEI) at the electrode/electrolyte interface substantially affects the stability and lifetime of lithium-ion batteries (LIBs). One of the methods to improve the lifetime of LIBs is by the inclusion of additive molecules to stabilize the SEI. To understand the effect of additive molecules on the initial stage of SEI formation, we compare the decomposition and oligomerization reactions of a fluoroethylene carbonate (FEC) additive on a range of oxygen functionalized graphitic anode to those of an ethylene carbonate (EC) organic electrolyte. A series of density functional theory (DFT) calculations augmented by ab-initio molecular dynamics (AIMD) simulations reveal that EC decomposition on an oxygen functionalized graphitic (1120) edge facet through an $\mathrm{S}_{\mathrm{N}} 2$ mechanism is spontaneous, even in an uncharged cell. Decomposition of EC through an $\mathrm{S}_{\mathrm{N}} 2$ reaction pathway results in alkoxide species regeneration which is responsible for continual oligomerization along the graphitic surface. In contrast, FEC prefers to decompose through an $\mathrm{S}_{\mathrm{N}} 1$ pathway, which does not promote alkoxide regeneration. The ability of FEC as an additive to suppress alkoxide regeneration results in a smaller and thinner SEI layer that is more flexible towards lithium intercalation during the charging/discharging process. In addition, the presence of different oxygen functional groups at the surface of graphite dictates the oligomerization products and $\mathrm{LiF}$ formation in the SEI.
\end{abstract}




\section{Introduction}

One of the main challenges in controlling the efficacy of lithium-ion batteries (LIBs) is the loss of cyclable lithium ions that results in capacity fade over extended use. Capacity fading observed in LIBs stems from the formation of a solid electrolyte interphase (SEI) at the electrode/electrolyte interface. ${ }^{1-3}$ SEI formation in LIBs is due to various side reactions between electrolyte species and the electrode surface that result in electrolyte decomposition. The accumulation of decomposed electrolyte on electrode surfaces blocks the lithium intercalation pathway and reduces the electrode's availability to cycle lithium, ${ }^{4}$ thus decreasing both the power and energy density of LIBs. ${ }^{5,6}$ In order to improve the lifetime of LIBs, a thorough understanding of electrode-electrolyte chemistry at the SEI is required to design better LIBs components. Although there have been significant studies on both electrodes and electrolyte materials separately, ${ }^{7-14}$ comprehensive knowledge of SEI formation remains elusive. The complexity of the interplay between the chemistries of many different components of the electrode and electrolyte at the interface makes the characterization of SEI difficult. ${ }^{15}$

Typically, LIB electrolytes are composed of organic electrolytes such as ethylene carbonate (EC), dimethyl carbonate (DMC), and diethyl carbonate (DEC) that are mixed with a tiny amount of salt (the most common salt being $\mathrm{LiPF}_{6}$ ). However, $\mathrm{LiPF}_{6}$ tends to undergo side reactions with organic electrolytes that result in its decomposition at the electrode/electrolyte interfaces. ${ }^{8,16,17}$ At the initial stage of cycling, the SEI at the anode surface is mainly composed of loosely held organic polymer compounds before transforming into a more compact structure of inorganic salts such as $\mathrm{Li}_{2} \mathrm{O}, \mathrm{Li}_{2} \mathrm{CO}_{3}$ and $\mathrm{LiF} .{ }^{18}$ At the electrolyte end, the SEI is capped by various carbonate based decomposition products such as $\mathrm{Li}_{2} \mathrm{CO}_{3}$, $\left(\mathrm{CH}_{2} \mathrm{OCO}_{2} \mathrm{Li}\right)_{2}$, and $\mathrm{ROCO}_{2} \mathrm{Li}^{4,7,19-21}$

It has been known that even small changes in the composition of either electrolytes or electrodes may alter the structure of an SEI and thus, its properties, significantly. For example, addition of small weight fractions of additives components such as fluoroethylene carbonate (FEC) $)^{22}$ and vinylene carbonate (VC) ${ }^{23}$ have been found to improve the stability of an SEI and are capable of extending both the performance and 
life cycle of LIBs. ${ }^{24-26}$ In comparison to organic electrolytes, the reduction potential of these additives are higher, which leads to easier reduction and decomposition on the surface of the anode. ${ }^{27-30}$ Oligomerization of additives results in the formation of polymeric species that modifies SEI composition, and changes both the structure and the mechanism by which it forms and grows. ${ }^{31}$

Decomposition of additives through oligomerization reactions involves nucleophilic alkoxides species. ${ }^{32,33}$ One of the most common alkoxide formation pathways involves the decarboxylation of a terminal carbonate group to form ethoxides. ${ }^{34}$ Oligomerization reactions are then initiated through nucleophilic attack by ethoxide at either the carbonyl carbon $\left(C_{C}\right)$ site via $S_{N} 1$ mechanism or one of the ethylene carbons $\left(\mathrm{C}_{\mathrm{E}}\right)$ of cyclic carbonate through $\mathrm{S}_{\mathrm{N}} 2$ mechanism. The difference in the nucleophilic attack sites of cyclic carbonate results in different decomposition products, thus affecting SEI composition. Attack on the $\mathrm{C}_{\mathrm{C}}$ site $\left(\mathrm{S}_{\mathrm{N}} 1\right.$ mechanism) yields polyethylene carbonate (PEC) and ethylene carbonate radical (EC•)/polyfluoroethylene carbonate (PFEC) as major decomposition products for EC and FEC, respectively. Meanwhile, attack on the $\mathrm{C}_{\mathrm{E}}$ site via $\mathrm{S}_{\mathrm{N}} 2$ mechanism yields polyethylene oxide (PEO) for $\mathrm{EC}$ and polyfluoroethylene oxide (PFEO) for FEC. ${ }^{35}$ Previous work included a reaction network analysis of EC and FEC decomposition in vacuum and confirmed that PEC and PEO are the most thermodynamically favorable products of EC decomposition via $\mathrm{S}_{\mathrm{N}} 1$ and $\mathrm{S}_{\mathrm{N}} 2$ mechanisms, respectively. ${ }^{36}$ In addition to oligomer species, decomposition through $\mathrm{S}_{\mathrm{N}} 2$ mechanism also releases gaseous $\mathrm{CO}_{2}$ which may then undergo further reaction with another $\mathrm{EC}$ molecule to form an inorganic $\mathrm{Li}_{2} \mathrm{CO}_{3}$ species. ${ }^{37-39}$ The oligomerization products obtained through $\mathrm{S}_{\mathrm{N}} 1$ and $\mathrm{S}_{\mathrm{N}} 2$ reaction pathways for both EC and FEC are summarized in Figure 1.
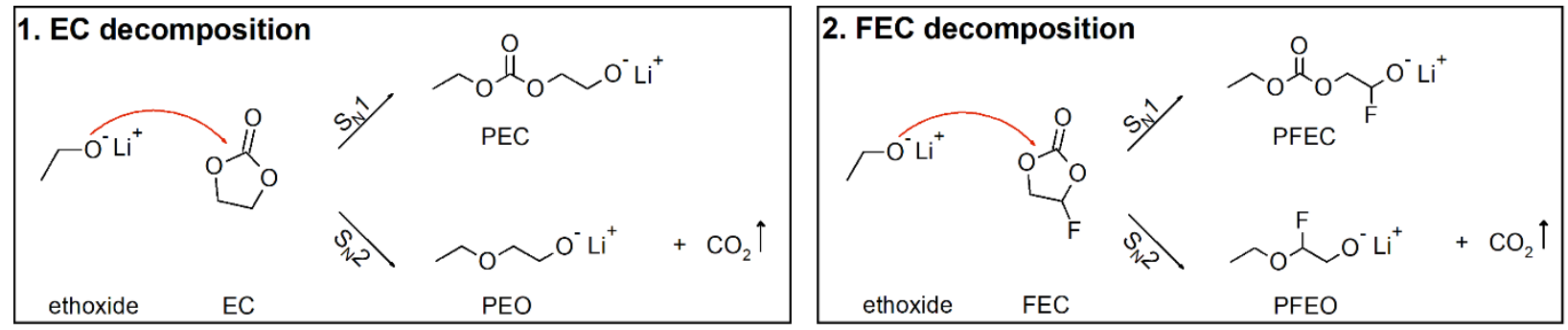

Figure 1: Oligomerization products of EC and FEC through $S_{N} 1$ and $S_{N} 2$ decomposition pathways. 
In vacuum, both $\mathrm{S}_{\mathrm{N}} 1$ and $\mathrm{S}_{\mathrm{N}} 2$ oligomerization pathways have been found to be exothermic. ${ }^{36}$ Coupled with $\mathrm{CO}_{2}$ generation, oligomerization through an $\mathrm{S}_{\mathrm{N}} 2$ pathway results in alkoxide regeneration, which is responsible for continual polymerization reactions along the electrode surface, as is observed in experiment. $^{34}$

In addition to the polarity of the electrolyte species, SEI formation is also found to be highly sensitive to the surface structure of electrodes. ${ }^{40}$ One of the most commonly used anode materials in LIBs is carbon based graphite, which may be functionalized by various oxygen functional groups such as hydroxyl $(-\mathrm{C}-\mathrm{OH})$, ketonic $(-\mathrm{C}=\mathrm{O})$, carboxyl $(-\mathrm{C}(-\mathrm{OH})=\mathrm{O})$, and carbonyl $(-\mathrm{C}(-\mathrm{H})=\mathrm{O})$ groups ${ }^{41}$ Surface functionalization helps to prevent graphite exfoliation, thereby bringing further stabilization to the anode. ${ }^{42}$ In battery studies, the effects of surface functionalization are often overlooked as the anode surface are quickly covered by the SEI after the initial cycling period. ${ }^{43}$ However, surface functionalization plays an important role at the initial stage of SEI formation, as it lowers the HOMO-LUMO bandgap between graphitic anode and isolated solvent molecules, ${ }^{43,44}$ thus increasing the reduction potential compared to $\mathrm{Li}^{+} / \mathrm{Li}$, which promotes faster electrolyte decomposition. ${ }^{45}$ Despite its significance in promoting electrolyte decomposition during initial cycling, the role of surface oxygenation in affecting the rate of formation and composition of SEI are yet to be known. Insights into the effect brought by different oxygenated surface functionalization groups on electrolyte decomposition, and thus, initial SEI formation are essential to guide modifications of LIB components to ultimately design batteries with longer lifetimes.

To better understand the initial SEI formation at the interface, decoupling of the effects brought by modifications to the electrolyte versus the electrode are imperative. The purpose of this study is to gain atomistic level insight into the effect brought by both oxygen functionalization of a virgin graphitic anode and FEC addition on initial SEI formation at the electrolyte/anode interface. To this end, we employ a series of first-principles density functional theory (DFT) calculations to study the reaction thermodynamics, charge distribution, and surface species evolution associated with decomposition of isolated EC and FEC 
molecules on five different graphitic surfaces: pristine, phenolic, ketonic, carboxyl, and carbonyl. The structural similarity between EC and FEC allows for direct comparison of decomposition reactions between the two molecules, which will highlight the effect of additives on the initial SEI formation.

The remainder of this paper is arranged as follows: First, we describe our atomistic models along with the methodology and computational details we used in our simulations. Then, we present our results of decomposition reaction thermodynamics and energy barriers, followed by analysis of charge transfer between functionalized graphitic anode and electrolyte molecules during decomposition reactions. We conclude by discussing the implications of our findings on the design of LIBs.

\section{Computational Methods}

\subsection{Model System}

The (1120) plane of graphitic armchair edge was used throughout our calculations rather than other edge terminations as out of all armchair and zigzag terminations, the (1120) armchair edge plane has the lowest surface energy and therefore is the most stable low-index edge terminated surface. ${ }^{46-48}$ Although the (0001) of the basal plane of graphite shows substantially lower surface energy than (1120) armchair edge, the superior stability of basal planes results in very unfavorable water decomposition thermodynamics. In contrast, water decomposition on the (1120) edge facet that results in oxygen functionalization of the graphitic surface is found to be spontaneous. ${ }^{46,49}$ The high reactivity of oxidized graphitic edge sites suggests that edge terminations are more favorable sites for electron transfer than the basal plane and are consistent with their known electrochemical activities. ${ }^{50}$ In battery studies, differences in chemical reactivity between different graphite terminations are often ignored as the anode surface are quickly covered by SEI after the initial cycling period. However, in the initial cycling period, the type of surface terminations plays a vital role in determining both the rate of SEI formation and its composition. ${ }^{43}$

Four layers of graphite with (11으) edge facets were fit into a simulation cell of $12.70 \times 13.54 \mathrm{x}$ $14.90 \AA^{3}$ subject to periodic boundary conditions, which were employed throughout all calculations. A 
vacuum gap of at least $10 \AA$ in the z-direction was incorporated to avoid spurious interactions between adjacent slabs. Five different functionalized graphitic surfaces were considered in our study: pristine graphite, hydroxyl $(-\mathrm{C}-\mathrm{OH})$, ketonic $(-\mathrm{C}=\mathrm{O})$, carboxyl $(-\mathrm{C}(-\mathrm{OH})=\mathrm{O})$, and carbonyl $(-\mathrm{C}(-\mathrm{H})=\mathrm{O})$. Incorporation of oxygen functionals on the surface of (1120) edge graphite surface was done through splitting of water molecules into different oxygen functionals that were adsorbed on the graphite surface as shown in Figure 2a)-d) to represent the hydroxyl-, ketonic-, carboxyl-, and carbonyl-terminated graphite surfaces, respectively. In order to obtain full surface coverage for our setup that consisted of 48 surface carbon atoms, different numbers of water molecules were required for different oxygen functionalization: 24, 16, 32 and 24 for phenolic, ketonic, carboxyl, and carbonyl, respectively. Figure 2e) shows the adsorption of polyfluoroethylene carbonate (PFEC) on the surface of the ketonic (1120) edge graphite as a representative of the simulation box used throughout this study. The model system was comprised of four graphitic layers, with water molecule was split into $-\mathrm{H},-\mathrm{O},-\mathrm{H}$ on the surface of pristine graphite to depict ketonic functionalization. 16 water molecules were required to decompose on the surface to obtain full surface coverage of ketonic functionalization.

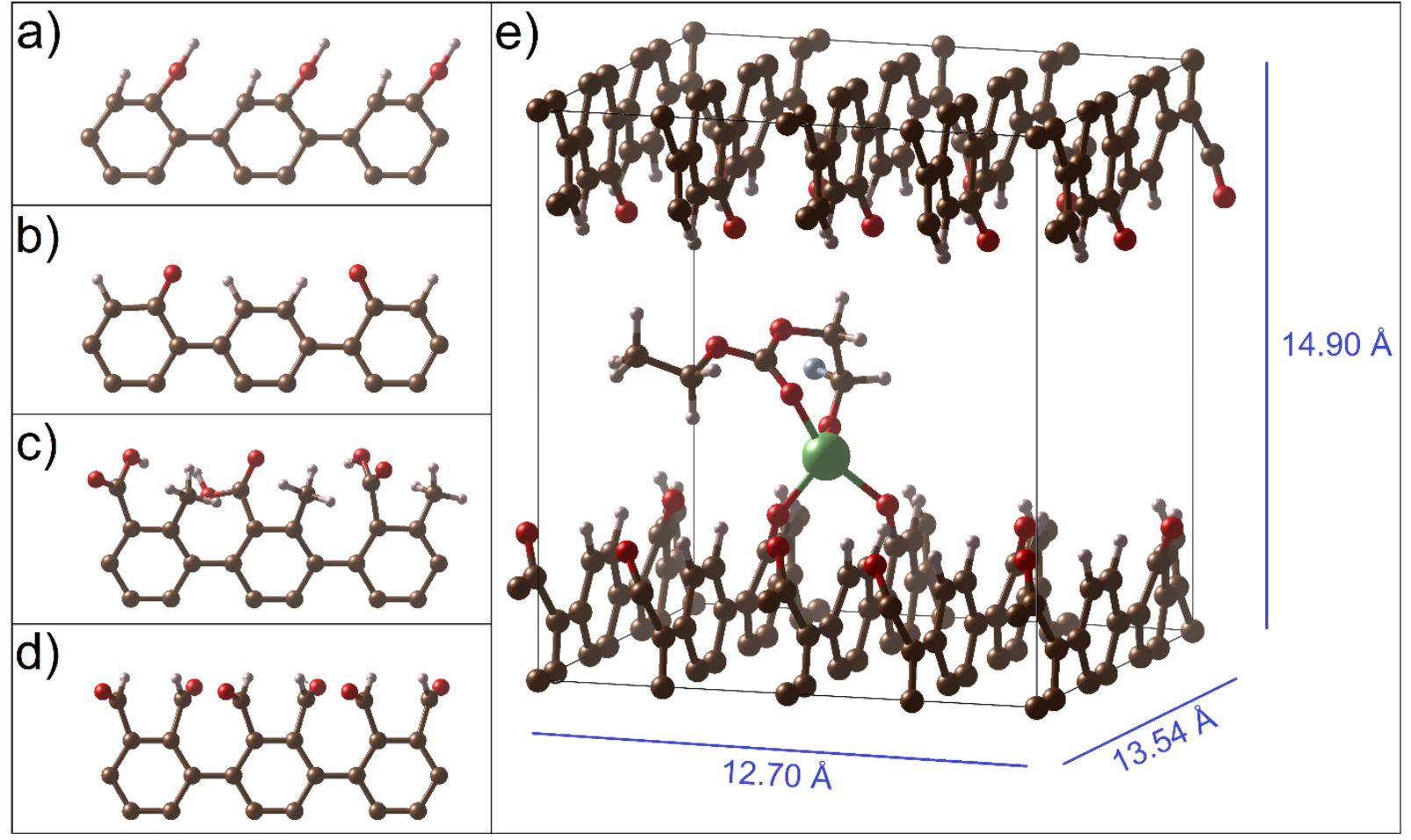


Figure 2: Oxygen functionalized edge (11무) graphite surfaces : a) hydroxyl-, b) ketonic-, c) carboxyl-, and d) carbonyl-terminated graphite surfaces, Figure 2e) shows the simulation cell used to model adsorption of polyfluoroethylene carbonate (PFEC) on ketonic edge (11 20$)$ graphite surface. $\mathrm{Li}, \mathrm{C}, \mathrm{O}, \mathrm{H}$, and $\mathrm{F}$ are depicted in green, brown, red, white, and purple, respectively.

To represent an uncycled virgin graphitic anode at the beginning of its first charging cycle, a graphitic anode was modeled without $\mathrm{Li}^{+}$ions. In addition, a neutral cell without application of an external field was utilized. We chose this setup for two reasons: 1) we were interested in the initial SEI formation at the very initial charging state before $\mathrm{Li}^{+}$ions intercalation on the anode takes place; and 2) application of an external field would have resulted in the distribution of excess electrons throughout the whole simulation cell rather than to be contained solely on the anode. The incorrect distribution of excess electrons has been argued in the literature to affect both the electronic and molecular distribution on the graphitic anode incorrectly. ${ }^{51}$

We also did not consider the presence of inorganic electrolytes such as $\mathrm{LiPF}_{6}$ in this study. Although $\mathrm{LiPF}_{6}$ has been known for its ability to attack and change the composition of an SEI, this study focused on the events that take place before the existence of an SEI at the interface. Additionally, it has been shown that $\mathrm{LiPF}_{6}$ molecules are located at the bulk end of electrolyte and are not able to penetrate to the electrode end of the SEI. ${ }^{51}$ Seeing that our study is focused on the reactions that take place at the surface of the electrode, it was justified that the presence of $\mathrm{LiPF}_{6}$ would not have affected the surface reactions significantly.

\subsection{Calculations Details}

All calculations were performed using the Vienna Ab initio Simulation Package (VASP) ${ }^{52,53}$ within the projector augmented-wave (PAW) approach. ${ }^{54}$ The generalized gradient approximation Perdew-Burke- 
Ernzerhof (GGA-PBE) functional ${ }^{55}$ was used in combination with DFT-D3 Grimme's empirical dispersion model to account for long-range dispersion interactions. ${ }^{56}$ Ionic cores of all atoms were described using the PAW potentials of $\mathrm{H}, \mathrm{C}, \mathrm{O}, \mathrm{F}$, and Li_sv that were chosen from the VASP PBE-PAW library. A planewave basis set cut-off energy was set to $500 \mathrm{eV}$. Atomic relaxation of graphite slabs was accomplished by fixing all but the two uppermost layers of carbon atoms to bulk positions, which were required to prevent C-C bond formations. ${ }^{47}$ Optimization convergence criteria for the two uppermost graphite layers were set to be $1 \times 10^{-5} \mathrm{eV}$ for electronic self-consistent iterations, and structures were adjusted until all the forces acting on ions were less than $0.02 \mathrm{eV} / \AA$. The 3 x 3 x 1 mesh of $k$-points in the Monkhorst-Pack scheme ${ }^{57}$ was chosen for the Brillouin zone sampling, and Gaussian smearing of $0.1 \mathrm{eV}$ was applied.

Adsorption configurations of EC and FEC and its decomposition products in accordance with the schematic given in Figure 1 on different functionalized graphitic slabs were probed separately through ab initio molecular dynamics (AIMD) simulations. The AIMD simulations were done at $\Gamma$-point with a time step of $\delta \mathrm{t}=1 \mathrm{fs}$ for at least $10 \mathrm{ps}$ until all the forces converged. The Nose-Hoover thermostat ${ }^{58,59}$ was employed to maintain the average temperature at $300 \mathrm{~K}$. The adsorption configurations for both reactant and product species on graphitic surface of different oxygen functionals that were obtained from AIMD simulations were further optimized using density functional theory (DFT) to calculate the reaction thermodynamics associated with EC and FEC surface decomposition. Saddle points along the reaction path was searched for using the climbing image nudged elastic band (CI-NEB) $\operatorname{method}^{60}$ to estimate the activation barriers associated with the decomposition of electrolyte molecules on graphitic surfaces. Electronic charge distribution and transfer between adsorbed molecule and graphitic surface was calculated according to the following equation: $\rho_{\text {difference }}=\rho_{\text {total }}-\rho_{\text {slab }}-\rho_{\text {electrolyte }},{ }^{51}$ which was visualized by using Visualization for Electronic and Structural Analysis (VESTA) software as an electron density difference $\operatorname{map}(\mathrm{EDDM}){ }^{61}$ 


\section{Results and Discussion}

The adsorption of ethoxide-Li-EC/FEC on the graphitic anode surface was found to be spontaneous, with three distinctive adsorption configurations subject to anode surface functionalization. Molecular dynamics (MD) simulations revealed that the adsorption of ethoxide-Li-EC/FEC on pristine graphite occurs through the ethoxide oxygen $\left(\mathrm{O}_{\mathrm{E}}\right)$ site, whereas the adsorption of ethoxide-Li-EC/FEC occurs through Li for all of the oxygen functionalized surfaces considered. Li was found to form two bonds with the hydroxyl and ketonic surfaces, which brings Li coordination to four. However, for the hydroxyl functionalized surface, one surface hydrogen atom is found to be released by the hydroxyl surface and binds to the oxygen of the ethoxide constituent of the ethoxide-Li-EC/FEC complex. Adsorption of the ethoxideLi-EC/FEC complexes on both carboxyl and carbonyl surfaces occur through formation of a bond between $\mathrm{Li}$ and a surface oxygen. Changing the electrolyte from EC to FEC did not affect the adsorption configurations of the complexes. The adsorption configurations of ethoxide-Li-EC/FEC complexes on pristine, hydroxyl, ketonic, carboxyl, and carbonyl surfaces are summarized in Figure 3.

a)

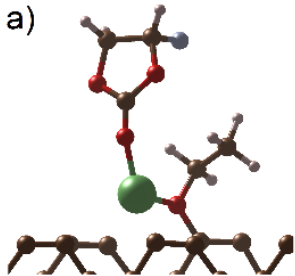

b)

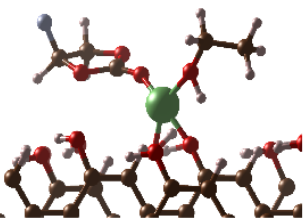

c)

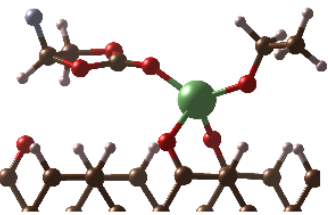

d)

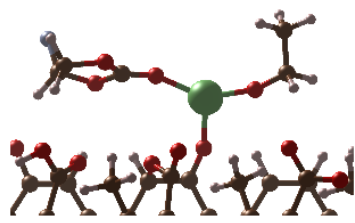

e)

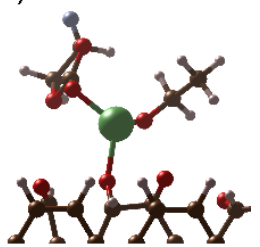

Figure 3: Ethoxide-Li-FEC complexes adsorption configurations on graphitic surfaces: a) pristine graphite, and b) hydroxyl-, c) ketonic-, d) carboxyl-, and e) carbonyl-terminated graphite. Adsorption configurations of ethoxide-Li-EC and ethoxide-Li-FEC complexes were found to be very similar and changing the electrolyte from EC to FEC did not affect the adsorption configuration of the complex on a particular graphitic surface. For both EC and FEC, one hydrogen atom is observed to be released by the hydroxyl surface, which then binds to the complexes through formation of an O-H bond with the ethoxide constituent of the complexes. Li, C, O, H, and F are depicted in green, brown, red, white, and purple, respectively. 
The reaction energies and barrier heights of $\mathrm{EC}$ and $\mathrm{FEC}$ decomposition reactions via $\mathrm{S}_{\mathrm{N}} 1$ and $\mathrm{S}_{\mathrm{N}} 2$ mechanisms on the graphitic anode of different surface oxygen functionalization obtained from static DFT and CI-NEB calculations are tabulated in Table 1.

\begin{tabular}{|c|c|c|c|c|c|c|c|c|}
\hline \multirow{4}{*}{$\begin{array}{l}\text { Surface } \\
\text { Termination }\end{array}$} & \multicolumn{4}{|c|}{$\mathrm{EC}$} & \multicolumn{4}{|c|}{ FEC } \\
\hline & \multicolumn{2}{|c|}{$\mathrm{S}_{\mathrm{N}} 1(\overline{\mathrm{eV}})$} & \multicolumn{2}{|c|}{$\mathrm{S}_{\mathrm{N}} 2(\mathrm{eV})$} & \multicolumn{2}{|c|}{$\mathrm{S}_{\mathrm{N}} 1(\mathrm{eV})$} & \multicolumn{2}{|c|}{$\mathrm{S}_{\mathrm{N}} 2(\mathrm{eV})$} \\
\hline & reaction & barrier & reaction & barrier & reaction & barrier & reaction & barrier \\
\hline & energies & heights & energies & heights & energies & heights & energies & heights \\
\hline pristine graphite & -0.36 & 3.36 & -4.56 & barrierless & -0.61 & 2.36 & 0.21 & 2.56 \\
\hline hydroxyl & -0.22 & 1.33 & -3.28 & barrierless & -0.65 & 0.13 & -0.59 & 1.01 \\
\hline ketonic & -2.61 & 0.86 & -2.68 & barrierless & -0.51 & 0.11 & -0.27 & 0.51 \\
\hline carboxyl & 1.43 & 1.78 & -1.42 & barrierless & 1.35 & 1.37 & 0.26 & 0.20 \\
\hline carbonyl & 7.30 & 7.48 & -7.98 & barrierless & 4.43 & 6.79 & -0.45 & 2.61 \\
\hline
\end{tabular}

Table 1: $S_{N} 1$ and $S_{N} 2$ reaction energies and barrier heights for ethoxide-Li-EC/FEC decomposition on graphitic (11ㅁㅇ) edge anode of different surface oxygen functionalization as obtained from static DFT and CI-NEB calculations, respectively.

A previous computational study from our group showed through reaction network analysis that PEC and PEO are the most thermodynamically favorable products of EC decomposition via $\mathrm{S}_{\mathrm{N}} 1$ and $\mathrm{S}_{\mathrm{N}} 2$ reaction pathways, respectively. ${ }^{36}$ As can be seen from Table 1, the $\mathrm{S}_{\mathrm{N}} 2$ oligomerization pathway is exothermic for all surfaces examined, while the $\mathrm{S}_{\mathrm{N}} 1$ pathway is only exothermic for pristine graphite, hydroxyl, and ketonic surfaces. The most exothermic $\mathrm{S}_{\mathrm{N}} 2$ decomposition for $\mathrm{EC}$ is on the carbonyl surface, which in combination with the highly endothermic $\mathrm{S}_{\mathrm{N}} 1$ reaction energies, implies that EC decomposition on the carbonyl surface would result in high PEO content. Although EC decomposition via an $\mathrm{S}_{\mathrm{N}} 1$ reaction pathway are also exothermic on both pristine graphite and hydroxyl surfaces, PEO would still be the major decomposition product on these surfaces as the reaction energies of $S_{N} 2$ mechanism are more exothermic than those of $S_{N} 1$ - provided that the reactions are limited by equilibrium thermodynamics. It can be concluded that in general, 
EC oligomerization on the oxygen functionalized graphitic (1120) edge surface favors an $\mathrm{S}_{\mathrm{N}} 2$ pathway except in the case of a ketonic surface. The small difference in the reaction energies between the two reaction pathways on the ketonic surface would result in a more equal mixture of PEC and PEO.

In contrast to $\mathrm{EC}$, the nature of the FEC decomposition pathway mechanism was found to be dependent on the type of oxygen functionalization on graphitic surface. FEC decomposition via $\mathrm{S}_{\mathrm{N}} 1$ mechanism is preferred on pristine graphite, while decomposition on a carbonyl surface prefers the $\mathrm{S}_{\mathrm{N}} 2$ reaction pathway. A carboxyl-terminated surface is less likely to promote FEC decomposition as both $\mathrm{S}_{\mathrm{N}} 1$ and $\mathrm{S}_{\mathrm{N}} 2$ reactions are endothermic. For FEC decomposition on a hydroxyl surface, the small difference in the reaction energies between $S_{N} 1$ and $S_{N} 2$ reaction pathways implies that both pathways are equally likely. The surface dependency observed for the FEC decomposition pathway thus suggests a more equally distributed mixture of oligomerization species instead of one major oligomerization product, as in the case for EC decomposition.

To get an insight into the competition between EC and FEC decomposition on a particular graphitic surface, we calculated the barrier heights for decomposition of both EC and FEC on all graphitic surfaces through CI-NEB. Table 1 shows that EC decomposition via $\mathrm{S}_{\mathrm{N}} 2$ mechanism is thermodynamically barrierless for all graphitic surfaces. For the $\mathrm{S}_{\mathrm{N}} 1$ decomposition pathway, FEC is found to have a lower reaction barrier than EC. Given the high ratio of EC:FEC molecules in the electrolyte, the oligomerization reaction at the electrolyte/graphitic anode interface during the initial SEI formation can be described as follows: First, EC would decompose spontaneously at the electrolyte/anode interface via $\mathrm{S}_{\mathrm{N}} 2$ mechanism. EC decomposition into polyethylene oxide (PEO) regenerates the ethoxide species which may attack another carbonate-based electrolyte and continue the oligomerization process along the surface of the graphitic anode. Eventually, ethoxide will react with FEC molecule. Nucleophilic attack of ethoxide on the FEC molecule results in aldehyde formation rather than ethoxide reformation, ${ }^{36}$ thus the presence of FEC molecules in the electrolyte terminates oligomer growth along the interface. The ability of FEC to suppress 
alkoxide regeneration reduces the size of oligomers in the SEI, which based on experimental studies, is responsible for the formation of a thinner and more flexible SEI layer. ${ }^{62,63}$

Adsorption configurations of oligomerization products PEC and PEO on graphitic surfaces are found to be similar to those of the ethoxide-Li-EC reactant complex shown in Figure 3. Both PEC and PEO prefer linear configurations on pristine, hydroxyl, and ketonic surfaces but prefer to adapt cyclic configurations on carboxyl and carbonyl surfaces. Figure 4 shows PEO configurations on different graphitic surfaces, which are analogous to the surface adsorption configurations of PEC.

a)

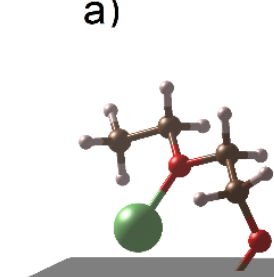

b)

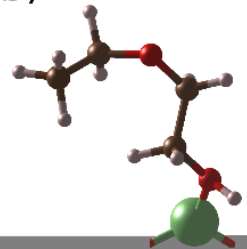

c)

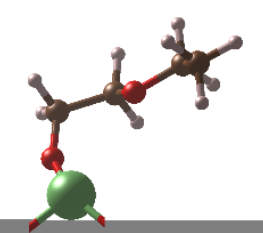

d)

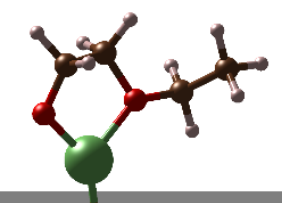

e)

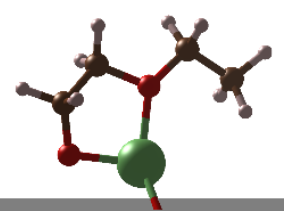

Graphitic Anode Bulk

Figure 4: PEO adsorption configurations on graphitic surfaces: a) pristine graphite, b) hydroxyl, c) ketonic, d) carboxyl, and e) carbonyl as main oligomerization product of EC molecule. PEC adsorption configurations are similar to those of $\mathrm{PEO}$ shown here. $\mathrm{Li}, \mathrm{C}, \mathrm{O}$, and $\mathrm{H}$ are depicted in green, brown, red, and white, respectively.

In the case of FEC decomposition and oligomerization, it has been widely acknowledged that the decomposition of FEC results in the F-abstraction by graphitic surfaces, which in the presence of Li ion promotes LiF deposition at the surface..$^{30,64-66}$ Many different reaction mechanisms have been proposed in the literature regarding LiF generation including: 1) alkoxide attack on FEC that yields $\mathrm{VC}$ and $\mathrm{LiF},{ }^{27,67}$ 2) multistep reaction involving one-electron ring-opening reduction of FEC followed by radical oligomerization of fluoropolymer which releases $\mathrm{LiF},{ }^{28,64,68}$ and 3) concerted decarboxylation and defluorination of FEC molecule that along with LiF also produces vinoxyl radical species ${ }^{35}$ However, there is still no consensus on the $\mathrm{LiF}$ formation reaction mechanism. 
To clarify the mechanism of LiF formation, we ran AIMD simulations for the FEC decomposition process through both $\mathrm{S}_{\mathrm{N}} 1$ and $\mathrm{S}_{\mathrm{N}} 2$ pathways on graphitic surfaces of different surface oxygen functionalizations. Our AIMD simulations show that FEC decomposition through $\mathrm{S}_{\mathrm{N}} 1$ mechanism results in the formation of EC radical $(\mathrm{EC} \bullet)$ through F-abstraction by pristine graphite, hydroxyl, and carboxyl surfaces. EC • prefers to adopt a linear configuration on surfaces that induce a more exothermic reaction (pristine graphite and hydroxyl), and to adopt bidentate configuration on the less reactive carboxyl surface. In contrast, FEC decomposition is not accompanied by F-abstraction on either ketonic or carbonyl surfaces, and PFEC oligomer remains as the main FEC decomposition product on these two surfaces. The absence of F-abstraction on both ketonic and carbonyl surfaces may stem from the lack of -OH group on the ketonic and carbonyl surfaces that serve as a source of proton to facilitate F-abstraction. Thus, in the presence of electropositive ions at the interface, such as $\mathrm{H}^{+}$or Li ion, F-abstraction may still occur. In comparison to the ketonic surface, F-abstraction by the carbonyl surface is more thermodynamically unfavorable (c.f. Table 1).

In the case of the formation of EC • on the pristine graphite surface, the F-abstraction causes electron transfer from the highly electronegative $\mathrm{F}$ atom to the graphite surface. The transferred electron density accumulates on the carbon atom next to $\mathrm{F}$ adsorption site. Adsorption of EC then occurs on this neighboring site through formation of a Li-C bond, with graphite surface acting as an electron donor. The Li facilitated adsorption implies that the pristine graphite surface now behaves similarly to the oxygen functionalized surfaces. EC • adsorption on the pristine graphite surface in the presence of a surface $\mathrm{F}$ is in contrast to all other adsorption on pristine graphite cases, where the pristine graphite serves as an electron acceptor and adsorption occurs through the ethylene oxygen $\left(\mathrm{O}_{\mathrm{E}}\right)$. Adsorption configurations and electronic distribution difference between ethoxide-Li-FEC and EC • radical on the pristine graphite surface are shown in Figure 5. 

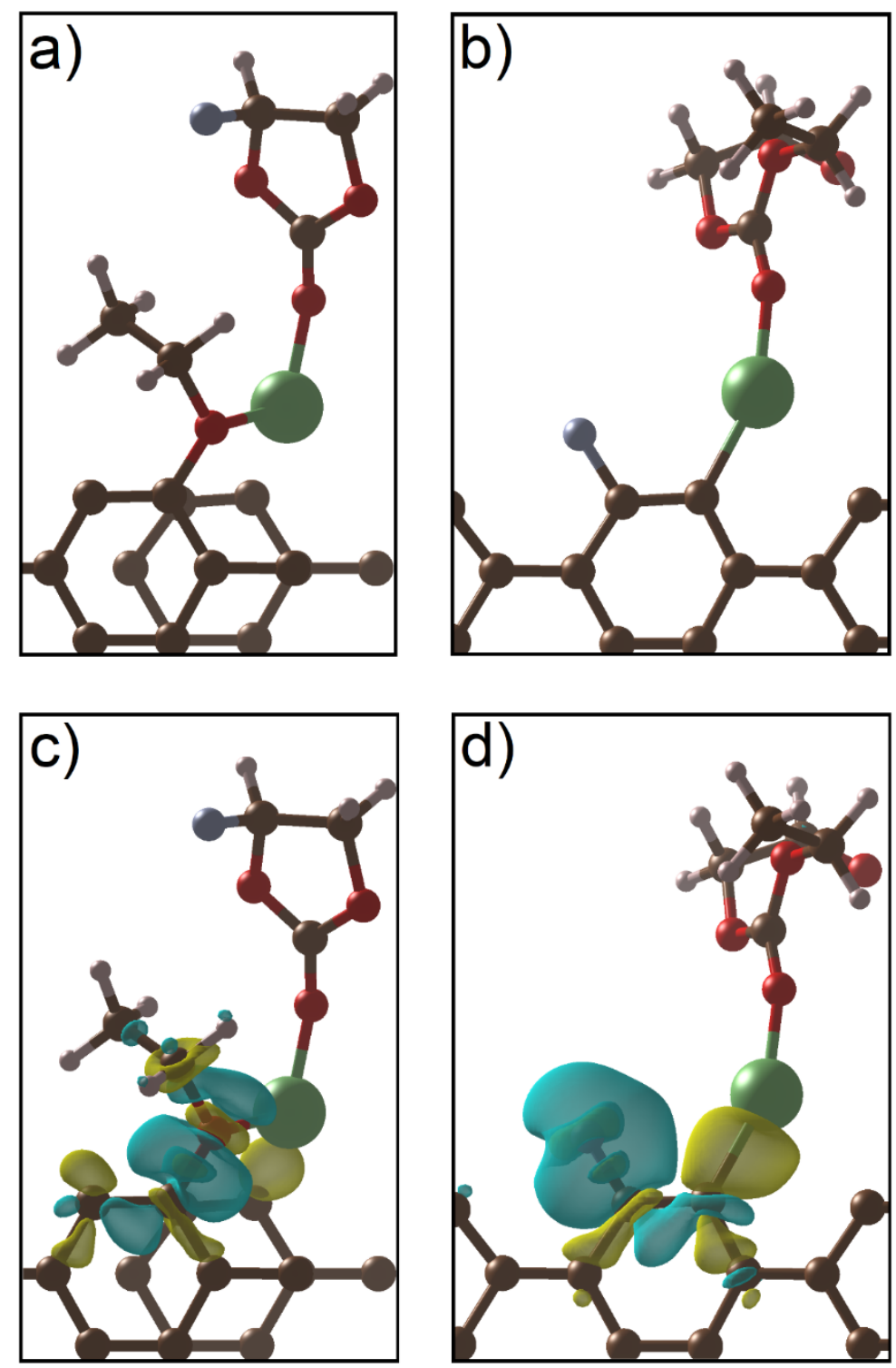

Figure 5: Adsorption configuration of a) ethoxide-Li-FEC and b) EC・ on pristine graphite (1120) surfaces; charge density difference distributions for c) ethoxide-Li-FEC and d) EC $\bullet$. Unit of charge density difference is e/Bohr. Yellow represents the electron accumulation area, while cyan is electron depletion area. Li, C, O, H, and F are depicted in green, brown, red, white, and purple, respectively.

Meanwhile, for FEC decomposition through an $\mathrm{S}_{\mathrm{N}} 2$ mechanism, we find that the coupling between decarboxylation and defluorination of the FEC molecule only happens on the hydroxyl surface. The Fabstraction by the hydroxyl surface leads to the formation of a vinoxyl radical. For all other surfaces 
considered in this study, FEC decarboxylation is not followed by spontaneous F-abstraction. In this case, the main product of the concerted FEC decomposition and decarboxylation is a PFEO oligomer rather than a vinoxyl radical. ${ }^{35}$ PFEO and vinoxyl radical are observed to prefer cyclic configurations on all surfaces but the ketonic surface. Although spontaneous F-abstraction was not observed on any surface except the hydroxyl surface for FEC $\mathrm{S}_{\mathrm{N}} 2$ decomposition, F-transfer may be induced through application of an external potential that would lower the LUMO of FEC to below the Fermi energy of the anode. ${ }^{69}$

Based on AIMD simulations, we conclude that the formation of LiF at the early stage of SEI in LIBs is subject to the ability of the anode surface to facilitate F-abstraction from FEC molecules. Pristine graphite, hydroxyl, and carbonyl surfaces are able to abstract the F atom from FEC, but ketonic and carbonyl surfaces do not. F-abstraction promotes the formation of radical species, of which is dictated by the type oxygen functional group present. F-abstraction by pristine graphite and carboxyl surfaces results in the formation of EC•, while hydroxyl surface may also produce vinoxyl radical in addition to $\mathrm{EC} \cdot$. A recent literature on lithium metal batteries also supports our finding of the relation between LiF formation and surface F-abstraction mechanism. ${ }^{70}$ This finding also consolidates the differences in LiF formation reaction mechanisms along with various oligomerization species detected in experiments: EC•, PFEC oligomer, vinoxyl radical, and PFEO. The chemical structure of EC•, PFEC oligomer, vinoxyl radical, and PFEO as products of FEC decomposition and oligomerization as obtained from our AIMD simulations are shown in Figure 6.

a)

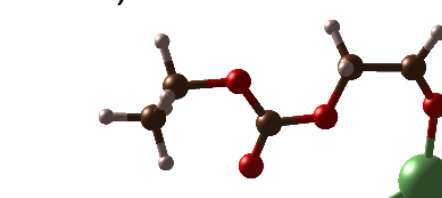

b)

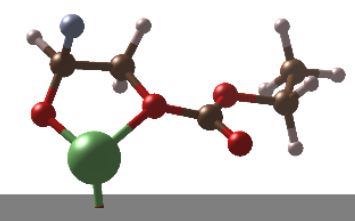

c)

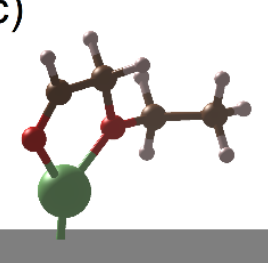

d)

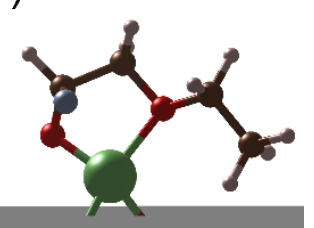
Graphitic Anode Bulk

Figure 6: FEC decomposition products as observed in AIMD simulations: a) EC • radical, b) PFEC oligomer, c) vinoxyl radical, and d) PFEO oligomer. In both a) and c), the F atom abstracted by the 
surface is not shown. For clarity, graphitic surfaces are simplified. $\mathrm{Li}, \mathrm{C}, \mathrm{O}, \mathrm{H}$, and $\mathrm{F}$ are depicted in green, brown, red, white, and purple, respectively.

\section{Conclusions}

We have investigated the effect of both FEC addition and oxygen functionalization on graphitic surfaces on the initial formation of an SEI at the uncharged virgin graphitic anode. AIMD simulations show that the alkoxide attack on the $\mathrm{C}_{\mathrm{C}}$ site of the $\mathrm{EC}$ molecule through $\mathrm{S}_{\mathrm{N}} 1$ reaction generates $\mathrm{PEC}$, while attacks on the $\mathrm{C}_{\mathrm{E}}$ site of the EC through $\mathrm{S}_{\mathrm{N}} 2$ pathway yield PEO and $\mathrm{CO}_{2}$. Oligomerization of FEC through $\mathrm{S}_{\mathrm{N}} 1$ results in a mixture of $\mathrm{EC} \cdot$ and $\mathrm{PFEC}$, while decomposition through $\mathrm{S}_{\mathrm{N}} 2$ yields formation of vinoxyl radical on the hydroxyl surface and PFEO on all other surfaces. A series of DFT calculations show that the $\mathrm{S}_{\mathrm{N}} 1$ reaction pathway favors FEC decomposition due to the greater reaction energies exothermicity and lower barriers for FEC decomposition than for EC on all graphitic surfaces. In contrast, EC oligomerization through $\mathrm{S}_{\mathrm{N}} 2$ reaction pathway into PEO is barrierless on all surfaces considered. The preference of FEC to undergo oligomerization through an $\mathrm{S}_{\mathrm{N}} 1$ pathway explains the ability of FEC to suppress regeneration of alkoxide, which is responsible for continual oligomerization along the surface of graphitic anode. This implies that the addition of FEC into the electrolyte mixture of LIBs results in a thinner, more flexible, and more superior SEI.

Variation of oxygen functionalization on graphitic surfaces affect the adsorption configurations of both reactant and products on the surfaces: through $\mathrm{O}_{\mathrm{E}}$ on pristine graphite, through formation of two Li-O bonds between the $\mathrm{Li}$ atom of EC/FEC complex and surface oxygen atoms on hydroxyl and ketonic surfaces, and in a monodentate fashion through one Li-O bond with surface oxygen on the carboxyl and carbonyl surfaces. FEC decomposition on pristine graphite and carboxyl leads to the formation of Fabstraction promotes the formation of $\mathrm{EC} \cdot$. In addition to $\mathrm{EC} \bullet$, the formation of vinoxyl radical is also observed on the hydroxyl surface. The ability of graphite surfaces to abstract F from FEC molecules 
correlates directly to the formation of $\mathrm{LiF}$. The relations between $\mathrm{LiF}$ formation, oligomerization products, F-abstraction mechanism, and surface oxygen functional groups on graphitic anode consolidates the differences in oligomerization products detected by experiments.

\section{Conflicts of Interest}

There are no conflicts to declare.

\section{Acknowledgements}

This research was entirely supported by the U.S. Department of Energy, Office of Science, Basic Energy Sciences, CPIMS Program, under Award DE-SC0019483. Computational resources for this work were provided by the Hyak supercomputer system of University of Washington and National Energy Research Scientific Computing Center (NERSC), a U.S. Department of Energy Office of Science User Facility operated under Contract No. DE-AC02-05CH11231. The authors would like to thank Dr. Stephanie Hare and Luke D. Gibson for helpful discussion and carefully reading this manuscript.

\section{References}

(1) Arora, P. Capacity Fade Mechanisms and Side Reactions in Lithium-Ion Batteries. J. Electrochem. Soc. 1998, 145, 3647-3667.

(2) Spotnitz, R. Simulation of Capacity Fade in Lithium-Ion Batteries. J. Power Sources 2003, 113, 7280 .

(3) Vetter, J.; Novák, P.; Wagner, M. R.; Veit, C.; Möller, K. C.; Besenhard, J. O.; Winter, M.; Wohlfahrt-Mehrens, M.; Vogler, C.; Hammouche, A. Ageing Mechanisms in Lithium-Ion Batteries. J. Power Sources 2005, 147, 269-281.

(4) Aurbach, D. A Comparative Study of Synthetic Graphite and Li Electrodes in Electrolyte Solutions Based on Ethylene Carbonate-Dimethyl Carbonate Mixtures. J. Electrochem. Soc. 1996, 143, 38093820 .

(5) Goodenough, J. B.; Park, K. S. The Li-Ion Rechargeable Battery: A Perspective. Journal of the American Chemical Society. 2013, pp 1167-1176.

(6) $\mathrm{Xu}, \mathrm{K}$. Nonaqueous Liquid Electrolytes for Lithium-Based Rechargeable Batteries. Chem. Rev. 2004, 104, 4303-4417.

(7) Xu, K.; Zhuang, G. V.; Allen, J. L.; Lee, U.; Zhang, S. S.; Ross, P. N.; Jow, T. R. Syntheses and Characterization of Lithium Alkyl Mono- and Bicarbonates as Components of Surface Films in LiIon Batteries. J. Phys. Chem. B 2006, 110, 7708-7719.

(8) Peled, E. An Advanced Tool for the Selection of Electrolyte Components for Rechargeable Lithium Batteries. J. Electrochem. Soc. 1998, 145, 3482-3486.

(9) Yamada, Y.; Iriyama, Y.; Abe, T.; Ogumi, Z. Kinetics of Lithium Ion Transfer at the Interface between Graphite and Liquid Electrolytes: Effects of Solvent and Surface Film. Langmuir 2009, 25, $12766-12770$.

(10) Xu, K.; Von Cresce, A.; Lee, U. Differentiating Contributions to "Ion Transfer" Barrier from Interphasial Resistance and $\mathrm{Li}+$ Desolvation at Electrolyte/Graphite Interface. Langmuir 2010, 26, $11538-11543$. 
(11) Tavassol, H.; Buthker, J. W.; Ferguson, G. A.; Curtiss, L. A.; Gewirth, A. A. Solvent Oligomerization during SEI Formation on Model Systems for Li-Ion Battery Anodes. $J$. Electrochem. Soc. 2012, 159, A730-A738.

(12) Porion, P.; Dougassa, Y. R.; Tessier, C.; El Ouatani, L.; Jacquemin, J.; Anouti, M. Comparative Study on Transport Properties for LiFAP and LiPF6 in Alkyl-Carbonates as Electrolytes through Conductivity, Viscosity and NMR Self-Diffusion Measurements. Electrochim. Acta 2013, 114, 95104.

(13) Park, Y.; Shin, S. H.; Hwang, H.; Lee, S. M.; Kim, S. P.; Choi, H. C.; Jung, Y. M. Investigation of Solid Electrolyte Interface (SEI) Film on LiCoO2 Cathode in Fluoroethylene Carbonate (FEC)Containing Electrolyte by 2D Correlation X-Ray Photoelectron Spectroscopy (XPS). J. Mol. Struct. 2014, 1069, 157-163.

(14) Möller, K. C.; Santner, H. J.; Kern, W.; Yamaguchi, S.; Besenhard, J. O.; Winter, M. In Situ Characterization of the SEI Formation on Graphite in the Presence of a Vinylene Group Containing Film-Forming Electrolyte Additives. J. Power Sources 2003, 119-121, 561-566.

(15) Heiskanen, S. K.; Kim, J.; Lucht, B. L. Generation and Evolution of the Solid Electrolyte Interphase of Lithium-Ion Batteries. Joule. 2019, pp 2322-2333.

(16) Peled, E. The Electrochemical Behavior of Alkali and Alkaline Earth Metals in Nonaqueous Battery Systems-The Solid Electrolyte Interphase Model. J. Electrochem. Soc. 1979, 126, 2047-2051.

(17) Aurbach, D.; Zinigrad, E.; Cohen, Y.; Teller, H. A Short Review of Failure Mechanisms of Lithium Metal and Lithiated Graphite Anodes in Liquid Electrolyte Solutions. Solid State Ionics 2002, 148, 405-416.

(18) Lu, P.; Li, C.; Schneider, E. W.; Harris, S. J. Chemistry, Impedance, and Morphology Evolution in Solid Electrolyte Interphase Films during Formation in Lithium Ion Batteries. J. Phys. Chem. C 2014, 118, 896-903.

(19) Shu, Z. X. Electrochemical Intercalation of Lithium into Graphite. J. Electrochem. Soc. 1993, 140, 922-927.

(20) Aurbach, D. The Correlation Between the Surface Chemistry and the Performance of Li-Carbon Intercalation Anodes for Rechargeable 'Rocking-Chair' Type Batteries. J. Electrochem. Soc. 1994, $141,603-611$.

(21) Nie, M.; Chalasani, D.; Abraham, D. P.; Chen, Y.; Bose, A.; Lucht, B. L. Lithium Ion Battery Graphite Solid Electrolyte Interphase Revealed by Microscopy and Spectroscopy. J. Phys. Chem. C 2013, 117, 1257-1267.

(22) McMillan, R.; Slegr, H.; Shu, Z. X.; Wang, W. Fluoroethylene Carbonate Electrolyte and Its Use in Lithium Ion Batteries with Graphite Anodes. J. Power Sources 1999, 81-82, 20-26.

(23) Aurbach, D.; Talyosef, Y.; Markovsky, B.; Markevich, E.; Zinigrad, E.; Asraf, L.; Gnanaraj, J. S.; Kim, H. J. Design of Electrolyte Solutions for Li and Li-Ion Batteries: A Review. In Electrochimica Acta; 2004; Vol. 50, pp 247-254.

(24) Jin, Y.; Kneusels, N. J. H.; Marbella, L. E.; Castillo-Martínez, E.; Magusin, P. C. M. M.; Weatherup, R. S.; Jónsson, E.; Liu, T.; Paul, S.; Grey, C. P. Understanding Fluoroethylene Carbonate and Vinylene Carbonate Based Electrolytes for $\mathrm{Si}$ Anodes in Lithium Ion Batteries with NMR Spectroscopy. J. Am. Chem. Soc. 2018, 140, 9854-9867.

(25) Jin, Y.; Kneusels, N. J. H.; Magusin, P. C. M. M.; Kim, G.; Castillo-Martínez, E.; Marbella, L. E.; Kerber, R. N.; Howe, D. J.; Paul, S.; Liu, T.; Grey, C. P. Identifying the Structural Basis for the Increased Stability of the Solid Electrolyte Interphase Formed on Silicon with the Additive Fluoroethylene Carbonate. J. Am. Chem. Soc. 2017, 139, 14992-15004.

(26) Ushirogata, K.; Sodeyama, K.; Okuno, Y.; Tateyama, Y. Additive Effect on Reductive Decomposition and Binding of Carbonate-Based Solvent toward Solid Electrolyte Interphase Formation in Lithium-Ion Battery. J. Am. Chem. Soc. 2013, 135, 11967-11974.

(27) Etacheri, V.; Haik, O.; Goffer, Y.; Roberts, G. A.; Stefan, I. C.; Fasching, R.; Aurbach, D. Effect of Fluoroethylene Carbonate (FEC) on the Performance and Surface Chemistry of Si-Nanowire Li-Ion Battery Anodes. Langmuir 2012, 28, 965-976. 
(28) Nakai, H.; Kubota, T.; Kita, A.; Kawashima, A. Investigation of the Solid Electrolyte Interphase Formed by Fluoroethylene Carbonate on Si Electrodes. J. Electrochem. Soc. 2011, 158, A798A801.

(29) McArthur, M. A.; Trussler, S.; Dahn, J. R. In Situ Investigations of SEI Layer Growth on Electrode Materials for Lithium-Ion Batteries Using Spectroscopic Ellipsometry. J. Electrochem. Soc. 2012, 159, A198-A207.

(30) Chen, X.; Li, X.; Mei, D.; Feng, J.; Hu, M. Y.; Hu, J.; Engelhard, M.; Zheng, J.; Xu, W.; Xiao, J.; Liu, J.; Zhang, J. G. Reduction Mechanism of Fluoroethylene Carbonate for Stable Solid-Electrolyte Interphase Film on Silicon Anode. ChemSusChem 2014, 7, 549-554.

(31) Zhang, S. S. A Review on Electrolyte Additives for Lithium-Ion Batteries. Journal of Power Sources. 2006, pp 1379-1394.

(32) Lee, J. C.; Litt, M. H. Ring-Opening Polymerization of Ethylene Carbonate and Depolymerization of Poly(Ethylene Oxide-Co-Ethylene Carbonate). Macromolecules 2000, 33, 1618-1627.

(33) Vogdanis, L.; Martens, B.; Uchtmann, H.; Hensel, F.; Heitz, W. Synthetic and Thermodynamic Investigations in the Polymerization of Ethylene Carbonate. Die Makromol. Chemie 1990, 191, 465472.

(34) Burkhardt, S. E. Impact of Chemical Follow-up Reactions for Lithium Ion Electrolytes: Generation of Nucleophilic Species, Solid Electrolyte Interphase, and Gas Formation. J. Electrochem. Soc. 2017, 164, A684-A690.

(35) Shkrob, I. A.; Wishart, J. F.; Abraham, D. P. What Makes Fluoroethylene Carbonate Different? J. Phys. Chem. C 2015, 119, 14954-14964.

(36) Gibson, L. D.; Pfaendtner, J. Solvent Oligomerization Pathways Facilitated by Electrolyte Additives During Solid-Electrolyte Interphase Formation. Phys. Chem. Chem. Phys. 2020, 22, 21494-21503.

(37) Schwenke, K. U.; Solchenbach, S.; Demeaux, J.; Lucht, B. L.; Gasteiger, H. A. The Impact of CO 2 Evolved from VC and FEC during Formation of Graphite Anodes in Lithium-Ion Batteries. $J$. Electrochem. Soc. 2019, 166, A2035-A2047.

(38) Xiong, D. J.; Ellis, L. D.; Nelson, K. J.; Hynes, T.; Petibon, R.; Dahn, J. R. Rapid Impedance Growth and Gas Production at the Li-Ion Cell Positive Electrode in the Absence of a Negative Electrode. $J$. Electrochem. Soc. 2016, 163, A3069-A3077.

(39) Broussely, M.; Biensan, P.; Bonhomme, F.; Blanchard, P.; Herreyre, S.; Nechev, K.; Staniewicz, R. J. Main Aging Mechanisms in Li Ion Batteries. In Journal of Power Sources; 2005; Vol. 146, pp 90-96.

(40) Boyer, M. J.; Vilčiauskas, L.; Hwang, G. S. Structure and Li+ Ion Transport in a Mixed Carbonate/LiPF6 Electrolyte near Graphite Electrode Surfaces: A Molecular Dynamics Study. Phys. Chem. Chem. Phys. 2016, 18, 27868-27876.

(41) Peled, E. Improved Graphite Anode for Lithium-Ion Batteries Chemically. J. Electrochem. Soc. 1996, 143, L4-L7.

(42) Yan, J.; Xia, B. J.; Su, Y. C.; Zhou, X. Z.; Zhang, J.; Zhang, X. G. Phenomenologically Modeling the Formation and Evolution of the Solid Electrolyte Interface on the Graphite Electrode for Lithium-Ion Batteries. Electrochim. Acta 2008, 53, 7069-7078.

(43) Leung, K.; Budzien, J. L. Ab Initio Molecular Dynamics Simulations of the Initial Stages of SolidElectrolyte Interphase Formation on Lithium Ion Battery Graphitic Anodes. Phys. Chem. Chem. Phys. 2010, 12, 6583-6586.

(44) An, S. J.; Li, J.; Daniel, C.; Mohanty, D.; Nagpure, S.; Wood, D. L. The State of Understanding of the Lithium-Ion-Battery Graphite Solid Electrolyte Interphase (SEI) and Its Relationship to Formation Cycling. Carbon 2016, 105, 52-76.

(45) Aurbach, D.; Teller, H.; Levi, E. Morphology/Behavior Relationship in Reversible Electrochemical Lithium Insertion into Graphitic Materials. J. Electrochem. Soc. 2002, 149, A1255-A1266.

(46) Jiang, Z.; Klyukin, K.; Alexandrov, V. Ab Initio Metadynamics Study of the VO2+/VO2+ Redox Reaction Mechanism at the Graphite Edge/Water Interface. ACS Appl. Mater. Interfaces 2018, 10, 20621-20626. 
(47) Thinius, S.; Islam, M. M.; Bredow, T. Reconstruction of Low-Index Graphite Surfaces. Surf. Sci. 2016, 649, 60-65.

(48) Incze, A.; Pasturel, A.; Chatillon, C. Ab Initio Study of Graphite Prismatic Surfaces. Appl. Surf. Sci. 2001, 177, 221-225.

(49) Ambrusi, R. E.; García, S. G.; Pronsato, M. E. Formation of Ag Nanowires on Graphite Stepped Surfaces. A DFT Study. Appl. Surf. Sci. 2015, 324, 710-717.

(50) McCreery, R. L. Advanced Carbon Electrode Materials for Molecular Electrochemistry. Chemical Reviews. 2008, pp 2646-2687.

(51) Qin, X.; Shao, M.; Balbuena, P. B. Elucidating Mechanisms of Li Plating on Li Anodes of LithiumBased Batteries. Electrochim. Acta 2018, 284, 485-494.

(52) Kresse, G.; Furthmüller, J. Efficiency of Ab-Initio Total Energy Calculations for Metals and Semiconductors Using a Plane-Wave Basis Set. Comput. Mater. Sci. 1996, 6, 15-50.

(53) Kresse, G.; Furthmüller, J. Efficient Iterative Schemes for Ab Initio Total-Energy Calculations Using a Plane-Wave Basis Set. Phys. Rev. B - Condens. Matter Mater. Phys. 1996, 54, 11169 11186.

(54) Blöchl, P. E. Projector Augmented-Wave Method. Phys. Rev. B 1994, 50, 17953-17979.

(55) Perdew, J. P.; Burke, K.; Ernzerhof, M. Generalized Gradient Approximation Made Simple. Phys. Rev. Lett. 1996, 77, 3865-3868.

(56) Grimme, S.; Antony, J.; Ehrlich, S.; Krieg, H. A Consistent and Accurate Ab Initio Parametrization of Density Functional Dispersion Correction (DFT-D) for the 94 Elements H-Pu. J. Chem. Phys. 2010, 132, 154104.

(57) Monkhorst, H. J.; Pack, J. D. Special Points for Brillouin-Zone Integrations. Phys. Rev. B 1976, 13, 5188-5192.

(58) Nosé, S. A Molecular Dynamics Method for Simulations in the Canonical Ensemble. Mol. Phys. 1984, 52, 255-268.

(59) Hoover, W. G. Canonical Dynamics: Equilibrium Phase-Space Distributions. Phys. Rev. A 1985, 31, 1695-1697.

(60) Henkelman, G.; Uberuaga, B. P.; Jónsson, H. Climbing Image Nudged Elastic Band Method for Finding Saddle Points and Minimum Energy Paths. J. Chem. Phys. 2000, 113, 9901-9904.

(61) Momma, K.; Izumi, F. VESTA: A Three-Dimensional Visualization System for Electronic and Structural Analysis. J. Appl. Crystallogr. 2008, 41, 653-658.

(62) Nie, M.; Demeaux, J.; Young, B. T.; Heskett, D. R.; Chen, Y.; Bose, A.; Woicik, J. C.; Lucht, B. L. Effect of Vinylene Carbonate and Fluoroethylene Carbonate on SEI Formation on Graphitic Anodes in Li-Ion Batteries. J. Electrochem. Soc. 2015, 162, A7008-A7014.

(63) Zhang, B.; Metzger, M.; Solchenbach, S.; Payne, M.; Meini, S.; Gasteiger, H. A.; Garsuch, A.; Lucht, B. L. Role of 1,3-Propane Sultone and Vinylene Carbonate in Solid Electrolyte Interface Formation and Gas Generation. J. Phys. Chem. C 2015, 119, 11337-11348.

(64) Nie, M.; Abraham, D. P.; Chen, Y.; Bose, A.; Lucht, B. L. Silicon Solid Electrolyte Interphase (SEI) of Lithium Ion Battery Characterized by Microscopy and Spectroscopy. J. Phys. Chem. C 2013, 117, 13403-13412.

(65) Choi, N. S.; Yew, K. H.; Lee, K. Y.; Sung, M.; Kim, H.; Kim, S. S. Effect of Fluoroethylene Carbonate Additive on Interfacial Properties of Silicon Thin-Film Electrode. J. Power Sources 2006, $161,1254-1259$.

(66) Kim, J. S.; Byun, D.; Lee, J. K. Electrochemical Characteristics of Amorphous Silicon Thin Film Electrode with Fluoroethylene Carbonate Additive. Curr. Appl. Phys. 2014, 14, 596-602.

(67) Zhao, X.; Zhuang, Q. C.; Xu, S. D.; Xu, Y. X.; Shi, Y. L.; Zhang, X. X. A New Insight into the Content Effect of Fluoroethylene Carbonate as a Film Forming Additive for Lithium-Ion Batteries. Int. J. Electrochem. Sci. 2015, 10, 2515-2534.

(68) Xu, C.; Lindgren, F.; Philippe, B.; Gorgoi, M.; Björefors, F.; Edström, K.; Gustafsson, T. Improved Performance of the Silicon Anode for Li-Ion Batteries: Understanding the Surface Modification Mechanism of Fluoroethylene Carbonate as an Effective Electrolyte Additive. Chem. Mater. 2015, 
27, 2591-2599.

(69) Borodin, O.; Ren, X.; Vatamanu, J.; Von Wald Cresce, A.; Knap, J.; Xu, K. Modeling Insight into Battery Electrolyte Electrochemical Stability and Interfacial Structure. Acc. Chem. Res. 2017, 50, 2886-2894.

(70) Zhang, Y.; Viswanathan, V. Not All Fluorination Is the Same: Unique Effects of Fluorine Functionalization of Ethylene Carbonate for Tuning Solid-Electrolyte Interphase in Li Metal Batteries. Langmuir 2020, 36, 11450-11466. 